\title{
Novo Coronavírus: Impacto na saúde mental dos profissionais de saúde
}

New Coronavirus: Impact on the mental health of health professionals

Nuevo coronavirus: Impacto en la salud mental de los profesionales de la salud

Geovanna Renaissa Ferreira Caldas

ORCID: https://orcid.org/0000-0001-9820-309X Centro Universitário de Juazeiro do Norte, Brasil

E-mail: geovannacaldas@hotmail.com

Lizandra Ellem Silva de Souza

ORCID: https://orcid.org/0000-0003-2398-325X Centro Universitário de Juazeiro do Norte, Brasil

E-mail: lizandraaellen@hotmail.com

Natalia Alves Nunes

ORCID: https://orcid.org/0000-0002-6667-760X Centro Universitário de Juazeiro do Norte, Brasil E-mail: natalia_nunes22@hotmail.com

Palomma Rafaelly Teixeira Alencar

ORCID: https://orcid.org/0000-0002-2261-4252

Centro Universitário de Juazeiro do Norte, Brasil E-mail: loma.cristo16@gmail.com

Katia Maria Gomes Brito

ORCID: https://orcid.org/0000-0002-5160-845X Universidade da Integração Internacional da Lusofonia Afro-Brasileira, Brasil E-mail: katia.britogomes@hotmail.com

Carla Carolina Alves Lopes

ORCID: https://orcid.org/0000-0002-8720-7738

Universidade Federal de São João Del Rei, Brasil E-mail: carla.lopes01@ outlook.com

Andrio Corrêa Barros

ORCID: https://orcid.org/0000-0002-2063-3177

Prefeitura Municipal de Primeira Cruz, Brasil E-mail: andriobarros@hotmail.com

Ana Caren dos Santos Paz

ORCID: https://orcid.org/0000-0002-2766-1357

Universidade Estadual do Maranhão, Brasil E-mail: ana.careen1@outlook.com

Adriana Soares Santos

ORCID: https://orcid.org/0000-0002-4956-1015

Faculdade Estácio São Luis Maranhão, Brasil

E-mail: adryannasoares353@gmail.com

Carolina Oliveira Lima Ferreira

ORCID: https://orcid.org/0000-0002-2859-8249 Universidade Federal da Bahia/ Faculdade de Medicina da Bahia, Brasil

E-mail: carolinal@ufba.br

Josefa Daiane dos Santos

ORCID: https://orcid.org/0000-0001-8796-4694

Universidade Tiradentes, Brasil

E-mail: daianeenf98@gmail.com

Ana Carolina Pinto da Silva

ORCID: http://orcid.org/0000-0002-9965-1772

Universidade Federal da Bahia, Brasil

E-mail: ana-carolina_pinto@hotmail.com

Miller Fontes Brandão

ORCID: https://orcid.org/0000-0002-4525-1845

Universidade Federal da Bahia, Brasil

E-mail: miller_brandao_100@hotmail.com

Samuel Melo Ribeiro

ORCID: https://orcid.org/0000-0003-2582-7883

Faculdade de Saúde e Ecologia Humana, Brasil

E-mail: samuelmeloribeiro@hotmail.com

Bianca Rodrigues Tavares

ORCID: https://orcid.org/0000-0002-7340-7309

Faculdade de Saúde e Ecologia Humana, Brasil

E-mail: biancatavares20@yahoo.com.br 


\title{
Mauro Marques Lopes \\ ORCID: https://orcid.org/0000-0001-6758-7844 Faculdade da Saúde e Ecologia Humana, Brasil E-mail: mauromllopes@gmail.com \\ Paulo Vitor Carvalho \\ ORCID: https://orcid.org/0000-0002-9924-3656 Faculdade Ciências Médicas de Minas Gerais, Brasil \\ E-mail: paulovitc@gmail.com \\ Fernanda de Oliveira \\ ORCID: https://orcid.org/0000-0001-7617-4910 \\ Faculdade da Saúde e Ecologia Humana, Brasil E-mail: fefe99oliveira@hotmail.com \\ Bruno Melo Ribeiro \\ ORCID: https://orcid.org/0000-0001-9975-4539 Faculdade Ciências Médicas de Minas Gerais, Brasil E-mail: brunomeloribeirob@hotmail.com \\ Nathália Carvalho \\ ORCID: https://orcid.org/0000-0001-7574-8548 Centro Universitário Presidente Tancredo de Almeida Neves, Brasil E-mail: nathcarv97@gmail.com
}

\begin{abstract}
Resumo
Objetivou-se descrever as evidências científicas sobre os sentimentos e agravos psicossociais entre os profissionais de saúde no enfrentamento da COVID-19. Trata-se de uma revisão integrativa, realizada nas bases de MEDLINE e LILACS, durante o período de maio e junho de 2020, utilizando os descritores em ciências da saúde e operador booleano: Covid-19 AND Saúde Mental AND Profissional, obtendo o total de 43 documentos, escolhidos 10 para revisão. Observamos que as medidas de isolamento e as demais estratégias tomadas para a prevenção e controle da doença tem causado diversos tipos de problemas na saúde mental dos profissionais que estão na linha de frente da pandemia, afetando a vida dessas pessoas. É possível concluir que o presente tema é de grande relevância pois os sentimentos negativos gerados pela doença podem acarretar problemas mentais mais graves sendo fundamental a busca de suporte psicológico para esses profissionais.
\end{abstract}

Palavras-chave: Covid-19; Saúde Mental; Pandemia.

\begin{abstract}
The objective was to describe the scientific evidence about psychosocial feelings and problems among health professionals in coping with COVID-19. It is an integrative review, carried out on the basis of MEDLINE and LILACS, during the period of May and June 2020, using the descriptors in health sciences and Boolean operator: Covid-19 AND Mental Health AND Professional, obtaining the total of 43 documents, chosen 10 for review. We observed that the isolation measures and other strategies taken for the prevention and control of the disease have caused several types of problems in the mental health of the professionals who are on the front line of the pandemic, affecting the lives of these people. It is possible to conclude that the present theme is of great relevance because the negative feelings generated by the disease can lead to more serious mental problems and it is essential to seek psychological support for these professionals.
\end{abstract}

Keywords: Covid-19; Mental health; Pandemic.

\section{Resumen}

El objetivo fue describir la evidencia científica sobre los sentimientos y problemas psicosociales de los profesionales de la salud en el afrontamiento del COVID-19. Se trata de una revisión integradora, realizada en base a MEDLINE y LILACS, durante el periodo de mayo y junio de 2020, utilizando los descriptores en ciencias de la salud y operador booleano: Covid-19 Y Salud Mental Y Profesional, obteniendo el total de 43 documentos, elegido 10 para revisión. Observamos que las medidas de aislamiento y otras estrategias tomadas para la prevención y control de la enfermedad han provocado diversos tipos de problemas en la salud mental de los profesionales que se encuentran en la primera línea de la pandemia, afectando la vida de estas personas. Es posible concluir que el presente tema es de gran relevancia ya que los sentimientos negativos que genera la enfermedad pueden ocasionar problemas mentales más graves, siendo fundamental la búsqueda de apoyo psicológico para estos profesionales.

Palabras clave: Covid-19; Salud mental; Pandemia.

\section{Introdução}

O novo tipo de coronavírus, denominado de SARS-CoV-2, apresenta-se com alta taxa de transmissibilidade e disseminação. Decorrente disso, na atualidade vem sendo vivenciado um momento de emergência de âmbito mundial, proporcionando um estado de estresse absoluto para a área da saúde (Shaw, 2020). 
Esse novo vírus foi identificado pela primeira vez na cidade de Wuhan, na China, o qual foi associado a um comércio local da cidade, que realizava a venda de animais vivos. Demandado a esse início, a propagação do vírus ocorreu de forma acelerada e sem controle pelos países, através da transmissão de pessoa para pessoa por meio do contato com aerossóis, gotículas, saliva, ou por objetos e superfícies que estivessem contaminadas (Lijun et al., 2020).

O paciente acometido pelo novo coronavírus pode apresentar quadro clínico com os sintomas característicos de uma síndrome gripal, caracterizado pela tosse, dispneia, falta de ar, febre, mialgia, fadiga, sintomas gastrointestinais e sintomas respiratórios superiores, até casos mais graves que apresentam episódios de choque séptico, insuficiência respiratória, síndrome da angústia respiratória aguda (SARA) e pneumonia. Deve-se considerar a possibilidade de pacientes assintomáticos (Brasil, Ministério da Saúde, 2020).

Em 06 de fevereiro de 2020, o Presidente da República do Brasil sancionou a Lei $\mathrm{n}^{\circ} 13.979$, que estabelece as medidas para enfrentamento da emergência de saúde, com foco na importância internacional decorrente da COVID-19. As recomendações sociais voltadas a população na tentativa de prevenir a transmissão da doença por intermeio da utilização do distanciamento social, sendo então à conduta mais rígida a ser aplicado e a principal medida adotada para o combate ao contágio, além da disponibilidade de testes laboratoriais e tratamentos médicos específicos embasados em protocolos que ainda passam por atualizações. É determinada a restrição da população em zonas sociais e proposto a se adequarem aos seus ambientes domiciliares, mantendo os hábitos de higiene como prevenção a disseminação do vírus (Brasil, Ministério da Saúde 2020).

A preocupação em não ser contaminado pela COVID-19 está presente em todos os momentos dos profissionais, no ambiente de trabalho ou no trajeto para o mesmo. Esse fato gera sentimento de insegurança dentro e fora do âmbito de trabalho. O efeito da pandemia tem mostrado a necessidade de investigar a importância da saúde metal em meio ao enfrentamento da COVID-19. (Faro et al., 2020).

Em geral, as preocupações de combate a pandemia estão voltadas para a área da saúde física da população, contudo, as medidas adotadas para contenção do vírus têm beneficiado uma parte e negligenciado outra. A enfermagem, durante sua atuação, têm sofrido sobrecarga, fadiga e preocupação em salvar vidas, com o grande fluxo de casos e o risco de contaminação, desencadeando um gatilho para os sintomas de estresse e ansiedade que podem evoluir para uma depressão (Schmidt et al., 2020).

Evidenciou-se que esses transtornos são desencadeados através da situação enfrentada atualmente, como o medo das consequências da doença, necessidade de distanciamento social, estresse devido à falta de rotina, questões financeiras abaladas, notícias de caráter falso ou notícias ruins constantes. (Duarte et al., 2020).

Este estudo tem como objetivo descrever as evidências científicas sobre os sentimentos e os agravos psicossociais apresentados entre os profissionais de saúde durante o enfrentamento da COVID-19.

\section{Metodologia}

Trata-se de uma revisão integrativa da literatura, de cunho descritiva e abordagem qualitativa, a qual segundo Mendes, Silveira \& Galvão (2019) pode ser descrita como uma pesquisa desenvolvida por intermeio da utilização de múltiplas pesquisas para possibilitar a síntese de conhecimento sobre um assunto, considerando 06 etapas: delimitar o tópico de interesse e a definição da pergunta; metodologia da amostragem, com a busca e seleção dos artigos, através da escolha dos critérios de inclusão e exclusão; extração dos dados; análise crítica dos dados selecionados; discussão sobre os resultados e exposição da revisão.

Para a obtenção dos dados, realizou-se a busca nas bases de dados Medical Literature Analysis and Retrieval Sistem Online e na Literatura Latino-Americana e do Caribe em Ciências da Saúde, com utilização dos Descritores em Ciências da 
Saúde: COVID-19; Saúde Mental e Profissional, cruzados com o operador booleano AND.

Entre os critérios de elegibilidade, foram incluídos: artigos originais que estivessem disponíveis na íntegra, com publicação nos idiomas português, inglês, chinês e espanhol, que abordassem a temática do estudo e que tivessem sido publicados entre os anos de 2019 a 2020. Como critérios de exclusão, cita-se: estudos repetidos, artigos que se apresentavam incompletos e outras revisões.

Através dos estudos selecionados, foram extraídas informações como: autor, ano, objetivo, amostra e os achados de cada artigo, separados e apresentados em dois quadros, onde utilizou-se a associação correspondente do número do artigo.

A busca resultou em uma totalidade de 43 documentos e após aplicação dos critérios previamente citados, restaram 38 estudos, desses, 33 encontravam-se na base de dados MEDLINE e 5 na base de dados LILACS, onde 35 foram produzidos na língua inglesa, 3 na língua portuguesa e 2 na língua espanhola.

\section{Resultados}

No intuito de contribuir e promover para o conhecimento voltado a temática central do estudo, foram analisados artigos que apresentaram o estado psicológico como foco, identificou-se que 10 produções abordavam a temática central desta revisão, analisando o que tem contribuído para as mudanças no hábito de vida dos profissionais para desenvolver problemas psicológico, conforme descritos nos quadros a seguir (Quadro 1):

Quadro 1: Caracterização da amostra.

\begin{tabular}{|c|c|c|c|}
\hline $\mathbf{N}^{\circ}$ & AUTOR/ANO & OBJETIVO & DESENHO \\
\hline 1 & $\begin{array}{l}\text { BLAKE et al., } \\
2020 .\end{array}$ & $\begin{array}{l}\text { Sintetizar informações para desenvolver e avaliar } \\
\text { um pacote de aprendizado digital para apoiar o } \\
\text { bem-estar psicológico de todos os profissionais de } \\
\text { saúde. }\end{array}$ & $\begin{array}{l}\text { Processo de três etapas, entre janeiro e março de } 2020 \text {, } \\
\text { realizado com cinco especialistas que desempenhavam papéis } \\
\text { estratégicos relacionados ao planejamento de saúde e bem- } \\
\text { estar dos funcionários da COVID-19. }\end{array}$ \\
\hline 2 & $\begin{array}{l}\text { DELGAGO et al., } \\
2020 .\end{array}$ & $\begin{array}{l}\text { Avaliar a realidade e as percepções sobre } \\
\text { segurança pessoal entre os profissionais de saúde } \\
\text { da América Latina. }\end{array}$ & $\begin{array}{l}\text { Estudo transversal, online, com base em pesquisa, } \\
\text { administrado por meio de questionário estruturado para } 936 \\
\text { profissionais de saúde na América Latina, de } 31 \text { de março de } \\
2020 \text { a } 4 \text { de abril de } 2020 \text {. }\end{array}$ \\
\hline 3 & $\begin{array}{l}\text { AHMED et al., } \\
2020 .\end{array}$ & $\begin{array}{l}\text { Avaliar a ansiedade e o medo de se infectar entre } \\
\text { os dentistas enquanto trabalhava durante o atual } \\
\text { surto de novas doenças por coronavírus (COVID- } \\
\text { 19). }\end{array}$ & $\begin{array}{l}\text { Estudo transversal, utilizando uma pesquisa on-line de } 10 \text { a } 17 \\
\text { de março de } 2020 \text {, com um total de } 669 \text { participantes de } 30 \\
\text { países diferentes em todo o mundo. }\end{array}$ \\
\hline 4 & $\begin{array}{l}\text { CUIYAN et al., } \\
2020 .\end{array}$ & $\begin{array}{l}\text { Entender os níveis de impacto psicológico, } \\
\text { ansiedade, depressão e estresse durante o estágio } \\
\text { inicial do surto de COVID-19. }\end{array}$ & $\begin{array}{l}\text { Pesquisa on-line usando técnicas de amostragem de bolas de } \\
\text { neve, incluindo } 1210 \text { entrevistados de } 194 \text { cidades da China. }\end{array}$ \\
\hline 5 & $\begin{array}{l}\text { BURHAN et al., } \\
2020 .\end{array}$ & $\begin{array}{l}\text { Avaliar o conhecimento dos especialistas e } \\
\text { residentes ao COVID-19 e suas atitudes em } \\
\text { relação aos pacientes infectados. }\end{array}$ & $\begin{array}{l}\text { Obtidos on-line entre } 13 \text { de março de } 2020 \text { e } 25 \text { de março de } \\
\text { 2020, por meio do site SurveyMonkey, com } 346 \text { especialistas e } \\
\text { residentes de várias instituições de saúde da Turquia, } \\
\text { utilizando um formulário de pesquisa. }\end{array}$ \\
\hline 6 & $\begin{array}{l}\text { JIANBO et al., } \\
2020 .\end{array}$ & $\begin{array}{l}\text { Avaliar a magnitude dos resultados em saúde } \\
\text { mental e fatores associados entre os profissionais } \\
\text { de saúde que tratam pacientes expostos ao } \\
\text { COVID-19 na China. }\end{array}$ & $\begin{array}{l}\text { Estudo transversal, estratificado por região com } 1257 \\
\text { profissionais de saúde em } 34 \text { hospitais de } 29 \text { de janeiro de } \\
2020 \text { a } 3 \text { de fevereiro de } 2020 \text { na China. }\end{array}$ \\
\hline
\end{tabular}




\begin{tabular}{|c|l|l|l|}
\hline 7 & JIAN et al., 2020. & $\begin{array}{l}\text { Chamar a atenção do público para a saúde mental } \\
\text { da equipe cirúrgica. }\end{array}$ & $\begin{array}{l}\text { Foram selecionados 120 indivíduos da equipe médica cirúrgica } \\
\text { de um Hospital em Xangai, preenchendo quatro escalas (escala } \\
\text { de ansiedade, escore de depressão, escore de ansiedade dos } \\
\text { sonhos e escala SF-36). }\end{array}$ \\
\hline 8 & YUAN et al., 2020. & $\begin{array}{l}\text { Comparar a frequência da Síndrome de Burnout } \\
\text { entre médicos e enfermeiros na linha de frente } \\
\text { (FL) e aqueles que trabalham em enfermarias } \\
\text { usuais }\end{array}$ & $\begin{array}{l}\text { Pesquisa com um total de 49 perguntas foi administrada a 220 } \\
\text { membros da equipe de saúde atuando contra o COVID-19. }\end{array}$ \\
\hline 9 & TAN et al., 2020. & $\begin{array}{l}\text { Examinar o sofrimento psicológico, depressão, } \\
\text { ansiedade e o estresse vivenciados pelos } \\
\text { profissionais de saúde em Cingapura no meio do } \\
\text { surto, e comparar entre o pessoal do hospital com } \\
\text { e sem treinamento. }\end{array}$ & $\begin{array}{l}\text { Realizado um questionário com escalas validadas de } \\
\text { depressão, ansiedade e estresse, entre fevereiro a março de } \\
\text { 2020 profissionais de saúde de 2 grandes instituições }\end{array}$ \\
\hline 10 & WEN et al., 2020. & $\begin{array}{l}\text { Avaliar o status psicológico dos profissionais de } \\
\text { saúde em todo o mundo. }\end{array}$ & $\begin{array}{l}\text { Pesquisa transversal com 2299 participantes por meio de } \\
\text { questionários on-line, com a escala de classificação numérica } \\
\text { (NRS) sobre o medo, a Hamilton Anxiety Scale (HAMA) e a } \\
\text { Hamilton Depression Scale (HAMD). }\end{array}$ \\
\hline
\end{tabular}

Fonte: Elaboração dos próprios autores.

Após a seleção dos 10 artigos que compuseram o seguinte estudo, através da caracterização da amostra com a identificação dos objetivos e a leitura desses documentos, possibilitou a criação de um quadro (Quadro 2) que abordasse especificamente as linhas de estudo dos artigos, categorizadas em sentimentos relatados dos profissionais, agravos psicológicos e sociais advindos da COVID-19, da atuação na linha de frente e da vivência perante uma pandemia, incluindo ainda possíveis estratégias de enfrentamento perante essas dificuldades.

Quadro 2: Linhas de estudo

\begin{tabular}{|c|c|c|}
\hline \multicolumn{3}{|c|}{ LINHAS DE ESTUDO } \\
\hline \multirow{4}{*}{ SENTIMENTOS } & MEDO & $01,02,03,05,08$ \\
\hline & PREOCUPAÇÃO & 04,08 \\
\hline & ESTRESSE & 01, 04, 07, 09 \\
\hline & ENFRENTAMENTO & 01,08 \\
\hline \multirow{3}{*}{ AGRAVOS PSICOSSOCIAIS } & ANSIEDADE & $01,03,04,06,07,09,10$ \\
\hline & INSÔNIA & 06 \\
\hline & DEPRESSÃO & $01,04,06,07,09,10$ \\
\hline \multirow{4}{*}{$\begin{array}{l}\text { ESTRATEGIA DE } \\
\text { ENFRENTAMENTO }\end{array}$} & CAPACITAÇÕES & 05,09 \\
\hline & INTERVENÇÕES PSICOLÓGICAS & 07,10 \\
\hline & AUTOCUIDADO & 01 \\
\hline & SEGURANÇA/ EPI & 02,05 \\
\hline
\end{tabular}

Fonte: Elaboração dos próprios autores. 


\section{Discussão}

\subsection{Isolamento Social}

Com a entrada da COVID-19 no Brasil, algumas táticas de restrição para o controle do vírus foram tomadas, visando à vigilância, o controle e o cuidado da doença, onde, de acordo com Bezerra et al (2020) as ações de assistência foram geradas pelas equipes locais de ações sanitárias nas diferentes esferas de governo.

Para Oliveira, Lucas \& Iquiapaza (2020) o coronavírus possui RNA positivo o que facilita a sua proliferação em diferentes ambientes, por ser uma nova composição identificada no amo de 2019, e tem como forma principal de contagio o contato direto a pessoas infectadas, por meio das vias de abertura (nariz, olhos e boca) possuído como principal medida, evitar a saída de casa e respeitando o isolamento social.

Dentre os possíveis planos diferentes que podem ser adotados entre as regiões, destaca-se como escolha frente ao momento atual, o isolamento social, como sendo a estratégia mais adotada para controle da doença. Essa medida foi inserida pela necessidade de reduzir significamente os riscos e probabilidades de infecção e dispersão do vírus (Faro et al., 2020). O isolamento também pode receber o nome de quarentena ou contenção comunitária, consistindo em uma separação de todas as pessoas, pelo risco de já ter tido contato com a doença (Schuchmann et al., 2020).

Ainda sobre a escolha do isolamento como principal medida para o enfrentamento da COVID-19, Fonseca et al (2020) destacam que este tipo de medida pode influenciar e interferir no processo de estado mental do dia-a-dia de crianças, adultos, idosos e principalmente dos profissionais da área da saúde, em decorrência da mudança repentina nas rotinas, no cotidiano, além das restrições de deslocamento entres os lugares. Ornell et al (2020) reforçam a ideia que o processo de se isolar causa grandes impactos no psicológico de toda a população.

No entanto, é importante ressaltar as principais medidas de isolamento social, que consiste em dois tipos, casos de pessoas que cumprem e seguem as diretrizes propostas e as que necessitam do isolamento domiciliar devido a contaminação, no entanto é importante ressaltar que o isolamento não é permitido nas devidas situações: Pessoas com idade superior a 65 anos ou menor de 2 anos, gestante e portadoras de doenças crônicas (Minas Gerais, 2020).

Seguindo a mesma linha de pesquisa dentre os critérios de grupos de risco para infecção, os impactos sofridos mentalmente em decorrência da necessidade de escolha desse tipo de medida de controle, Hammerschmidt \& Santana (2020) evidencia o fato de que as pessoas idosas são mais vulneráveis a doença e aos impactos psicológicos, apresentando então, um maior número de óbitos pelo fato de comorbidades e idade avançada, intensificando o isolamento.

\subsection{Sentimentos e Agravos Psicossociais}

Rodrigues \& Silva (2020), expõem a pandemia como uma forte batalha que os profissionais da saúde estão lidando, dando um enfoque para a atuação que está sendo realizada pelos profissionais da área da enfermagem, pois são os profissionais que estão trabalhando na linha de frente, ou seja, diretamente com os pacientes com suspeita e/ou pacientes confirmados com o vírus do novo COVID-19.

Vasconcelos et al (2020) explicam que esse acelerado avanço da doença provocou o aparecimento de muitas informações que podem proporcionar e impactar mudanças nos comportamentos das pessoas, levando-as a um adoecimento mental, originando sensações de medo, ansiedade e diversas inseguranças que poderão ser observadas até mesmo depois que o vírus já estiver controlado.

Segundo Mauch et al (2020), existem vários problemas causados pela COVID-19, gerados de forma direta ou indireta, afetando os aspectos econômicos, físicos, biológicos, sociais e psicológicos, ocasionando aumento da ansiedade e do estresse em pessoas que anteriormente apresentavam-se com a sua saúde mental estável. Em pessoas que tenham ou já tiveram em algum momento da vida qualquer tipo de problema voltado para a saúde mental, esses sintomas podem ser reforçados. 
Yuan et al (2020), informam que os profissionais que se encontram em trabalho continuo nas suas unidades, estavam extremamente preocupados consigo mesmo ou com algum membro da família terem sido infectados e também temem que a crise da COVID-19 continue por um longo intervalo de tempo. Baseado na mesma ideia, Burhan et al (2020) descrevem essa mesma afirmação, evidenciando ainda, patologias advindas como consequências dessas preocupações, sendo que mais da metade dos enfermeiros relataram sintomas de depressão (50,4\%), ansiedade (44,6\%), insônia (34,0\%) e angústia (71,5\%).

Seguindo a mesma linha de pesquisa, Ahmed et al (2020), Cuiyan et al (2020), Wen et al (2020), Jianbo et al (2020), Jian et al (2020) e Tan et al (2020), evidenciam dados sobre a incidência e prevalência de agravos psicossociais, como a ansiedade, depressão, insônia, medo, angustia e estresse nesses profissionais, em consequência do medo de contaminação própria, de alguém da família ou de amigos, principalmente os enfermeiros que se encontram como real linha de frente do cuidado.

O aumento repentino dos casos confirmados também trouxe enorme impactos no estresse e na ansiedade dos profissionais que atuavam nas equipes cirúrgicas da linha de frente (Jian, et al., 2020).

Confirmando essa informação, Miranda et al (2020) apresentam os sentimentos e as sensações vivenciadas por essas pessoas, citando raiva, medo, angustia, inquietação, preocupação e a percepção de incapacidade perante a prática do cuidado, por consequências da situação de isolamento, as mudanças drásticas das atividades do dia a dia, as condições econômicas não serem suficientes para o próprio sustento e/ou sustento da família, além da sensação de não saber o que vai acontecer ou quando a situação irá melhorar.

A ansiedade pode ser descrita como um Transtorno Mental diretamente associado ao medo, perturbações e preocupações excessivas, gerando uma agitação de forma antecipada a algo que ainda está para acontecer. Dessa forma, como consequência da pandemia, está sendo vivenciado uma situação de dúvidas constantes, sobre a doença, transmissão e reinfecção, ausência de uma terapêutica de imunização, incertezas sobre o retorno da vida pré-COVID 19 e sobre as consequências do pós-COVID 19 (Rolim, Oliveira \& Batista, 2020).

O temor de ser contaminado pela COVID-19, que é um vírus que pode levar a fortes complicações e ao óbito, possuindo grande capacidade de transmissão e sendo pouco conhecido atualmente, com muitas incertezas e ainda poucas evidências cientificas sobre a COVID-19. Tal situação afeta a saúde mental das pessoas, causando sentimento de insegurança, ansiedade que muitas vezes, leva a um quadro de depressão agravada ao decorrer da pandemia. (Ribeiro et al., 2020).

Os profissionais de enfermagem no cotidiano de trabalho passam por situações que acaba acarretando aumento da ansiedade e estresse, levando ao adoecimento mental e principalmente ao esgotamento profissional causando depressão e afetando totalmente a vida dessas pessoas. A falta de material para proteção individual é um dos motivos de preocupação desses profissionais pois sem eles o risco de contaminação é maior o que leva ao sentimento de medo e desanimo para concluir suas atividades (Humerez, Ohl \& Silva, 2020).

O trabalho do enfermeiro que está na linha de frente da COVID-19 é feito em um meio que é carregado de momentos que exige completamente do profissional que tem que lhe dar com a aflição, dor, inquietação, queixas e inseguranças dos pacientes, onde muitas vezes tem óbitos além da falta de equipamentos necessários para o cuidado a essas pessoas o que os levam a ter sentimentos de insatisfação, estresse e incapacidade causando desanimo que podem gerar um quadro grave de adoecimento mental (Pereira et al., 2020).

Podemos observar que por conta de todo movimento sobre a ação dos enfermeiros frete a pandeia causada pela COVID-19, houve uma relevância sobre as noites mal dormidas. Com o excesso de trabalho muitos profissionais apresentam insônia, seja por preocupações com seus familiares por conta das contaminações ou pela sobrecarga dos cuidados intensivos no local de trabalho. Logo percebemos que a COVID-19 traz consigo não apenas medo sobre suas ocorrências, porem também problemas psicológicos relacionados ao sono (Pereira et al., 2020). 


\subsection{Estratégias de Enfrentamento}

Delgado et al (2020) apresentam um estudo sobre a sensação de segurança dos profissionais de saúde, dos quais, muitos descreveram que não tiveram acesso a alguma política e/ou procedimentos voltados para a sua própria segurança. Em decorrência disso, gerou-se medo e insegurança nos profissionais durante a realização da assistência aos pacientes suspeitos ou confirmados. Os profissionais da saúde, que por muitas vezes não tem alternativas de escolha acabam trabalhando em ritmo mais pesado, com maiores cargas horarias e maiores responsabilidades, além das pré-existentes, além de correrem o risco da contaminação. Descobrindo como únicas estratégias adotadas para sua segurança, são os cuidados advindos de outros funcionários da instituição, como a higienização das paredes e cadeiras, limpeza das salas e objetos e os cuidados advindos da própria unidade, como esterilizações dos matérias e disponibilização dos equipamentos de proteção individual (EPI'S).

A doença (COVID-19) tem se avançado rapidamente e o pico de contaminação foi entre Junho/julho, o que está por vim agora é os cuidados para não ocorrer a contaminação por aglomerações, tendo em vista os cuidados redobrados evitando desta forma aglomerações desnecessárias. Na tentativa de realizar estratégias de apoio para a equipe de enfermagem, Blake et al (2020) descrevem que os líderes podem e devem buscar ideias alternativas para proporcionar algum tipo de auxílio para os profissionais atuantes, podendo ocorrer através de orientações, informações, capacitações, um local de trabalho e pausa dignos, momentos para descanso e auxilio no enfretamento de todas as sensações.

Dessa forma, é necessário buscar formas de encontrar ou manter um bom nível de equilíbrio do emocional, através de certos manejos e técnicas terapêuticas voltadas para o psicológico. Ribeiro, Souza \& Eler (2020) citam algumas estratégias que podem ser utilizadas no desenvolvimento da estabilização da saúde mental, como o não isolamento total, mantendo algum tipo de contato com familiares ou amigos, mesmo que seja com o uso de tecnologias, além da importância de criar rotinas e hábitos de vida saudável, identificar e/ou escolher novos hábitos para distrações nos momentos que estiver em casa e buscar redes de apoio online.

Uma das alternativas que pode ser considerada é a Terapia Cognitivo-Comportamental (TCC), consistindo em uma psicoterapia que baseia-se na ideia do indivíduo estrutura suas experiências do dia a dia afetam e determinam o seu comportamento, ações e sentimentos. Explicando como as situações não geram sentimentos ruins, mas sim, como as pessoas entendem e interpretam essas ocorrências. Sendo então, uma terapêutica extremamente efetiva para a redução de impactos psicológicos, decorrentes de situações como os sentimentos advindos do isolamento e do COVID-19 (Reyes \& Fermann, 2017).

Segundo Zanon et al (2020) outra opção de terapêutica perante tais necessidades é a Psicologia positiva. Podendo ser descrita como uma área da psicologia que estuda as experiências positivas e subjetivas do ser humano, traços que são individuais e positivos e instituições positivas, buscando assim, prevenir o máximo possível de patologias e adoecimentos mentais, através de uma prática baseada em evidências, auxiliando a enfrentar situações de estresse e ensinando a como lidar com as situações-problemas evidenciadas.

\section{Considerações Finais}

Em decorrência do momento vivenciado, os profissionais da área da saúde convivem com uma situação estressante, que gera incerteza sobre o seu próprio futuro e o futuro dos familiares, amigos, colegas de trabalho, com forte risco de sofrer adoecimento físico ou mental, com grandes impactos no psicológico.

Dentre os principais transtornos mentais desenvolvidos pelos profissionais da saúde, em especial pela equipe de enfermagem se encontra a ansiedade, insônia, angustia e medo. Tais sentimentos são eixos centrais de diversos estudos, que os apontam como gatilhos para o desenvolvimento de maiores agravos mentais como a depressão, além de impactos a saúde em seus mais amplos aspectos. 
Com base nas estratégias analisadas propõem-se implementação de medidas efetivas e de caráter emergencial, como a melhoria de suporte e apoio psicológico com acolhimento e escuta qualificada para os profissionais da saúde, utilizando de técnicas da psicologia para a diminuição do sofrimento mental dessas pessoas que compõem a linha de frente de cuidado aos pacientes com suspeita ou diagnosticados com COVID-19.

As ofertas de treinamentos e capacitações para as equipes que auxiliam na saúde mental desses profissionais também podem contribuir de forma significativa na melhoria da saúde dessas pessoas. Carecendo assim, de um olhar especial dos chefes, gestores e do Ministério da Saúde para os seus próprias profissionais, que ao cuidarem do outro, estão se desgastante no físico e no emocial, sofrendo danos e adquirindo sentimentos que muitas das vezes evoluem para algumas doenças/transtornos mentais.

Como sugestões para trabalhos futuros, indica-se a aplicação dessas estratégias de enfrentamento, para assim, poder observar se resultam em implicações positivas para a saúde mental dos profissionais da área da saúde, podendo ainda, identificar qual estratégia demonstrou melhor benefícios e se teve alguma que não gerou melhora.

\section{Referências}

Ahmed, M.A., et al. (2020). Fear and Practice Modifications among Dentists to Combat Novel Coronavirus Disease (COVID-19) Outbreak. International Journal Environ Research and Public Health, 17(8), 2821.

Bezerra, A.C.V., et al. (2020). Fatores associados ao comportamento da população durante o isolamento social na pandemia de COVID-19. Ciência \& Saúde Coletiva, 25, Rio de Janeiro.

Blake, H., et al. (2020). Mitigating the Psychological Impact of COVID-19 on Healthcare Workers: A Digital Learning Package. International Journal Environ Research and Public Health, 17(9), 2997.

Burhan, D,. et al. (2020). Attitudes of Anesthesiology Specialists and Residents toward Patients Infected with the Novel Coronavirus (COVID-19): A National Survey Study. Infecções Cirúrgicas, 21(4), 350-356.

Brasil, Ministério da Saúde. (2020). Lei no 13.979, de 6 de fevereiro de 2020. Dispõe sobre as medidas para enfrentamento da emergência de saúde pública de importância internacional decorrente do coronavírus responsável pelo surto de 2019. Diário Oficial da União, Brasília, Poder executivo, Brasília, DF, 06 fev 2020. Ed 27, seção 01, p 01.

Cuiyan, W., et al. (2020). Immediate Psychological Responses and Associated Factors during the Initial Stage of the 2019 Coronavirus Disease (COVID-19) Epidemic among the General Population in China. International Journal Environ Research and Public Health, 17(5), 1729.

Delgado, D., et al. (2020). Personal Safety during the COVID-19 Pandemic: Realities and Perspectives of Healthcare Workers in Latin America. International Journal Environ Research and Public Health, 17(8), 2798.

Duarte, M.Q., et al. (2020). COVID-19 e os impactos na saúde mental: uma amostra do Rio Grande do Sul. Revista Ciência \& Saúde Coletiva, 25(6).

Faro, A., et al. (2020). COVID-19 and mental health: The emergence of care. Contribuições da Psicologia no contexto da pandemia da COVID-19. Estudos de Psicologia., Campinas, 37, e.200074.

Fonseca, R.P.O., et al. (2020). Da proximidade ao distanciamento social: Desafios de sustentar a lógica da atenção psicossocial em tempos de pandemia. Health Residencies Journal-HRJ, 01(1), 48-64.

Hammerschmidt, K.S.A. \& Santana, R.F. (2020). Saúde do idoso em tempos de pandemia COVID-19. Cogitare Enfermagem, $25(1)$, e.72849.

Humerez, D.C., Ohl, R.I.B. \& Silva, M.C.N. (2020). Saúde mental dos profissionais de enfermagem do Brasil no contexto da pandemia COVID-19: Ação do Conselho Federal de Enfermagem. Cogitare Enfermagem, 25, e.74115.

Jianbo, L., et al. (2020). Factors associated with mental health outcomes among health care workers exposed to Coronavirus disease 2019. JAMA Network Open, 03(3), e.203976.

Jian, X., et al. (2020). Psychological status of surgical staff during the COVID-19 outbreak. Psychiatry Research, 288 , e.112955.

Lijun, K., et al. (2020). The mental health of medical workers in Wuhan, China dealing with the 2019 novel coronavirus. The Lancet Psychiatry, 07(14).

Mauch, A.G.D., et al. (2020). A utilização das redes sociais digitais no cuidado psicossocial infantojuvenil, diante da pandemia por Covid-19. Health Residencies Journal-HRJ, 01(2), 44-61.

Mendes, K.D.S., Silveira, R.C.C.P. \& Galvão, C.M. (2019). Uso de gerenciador de referências bibliográficas na seleção dos estudos primários em revisão integrativa. Texto contexto - enfermagem, 28, e.20170204.

Minas gerais. (2020). Governo do estado de Minas Gerais secretaria de estado de saúde. Plano estadual de contingência para emergência em saúde pública. Infecção humana pelo sars-cov-2 (Doença pelo coronavirus - covid-2019). 
Research, Society and Development, v. 10, n. 5, e33910515011, 2021

(CC BY 4.0) | ISSN 2525-3409 | DOI: http://dx.doi.org/10.33448/rsd-v10i5.15011

Miranda, F.M.D’A., et al. (2020). Condições de trabalho e o impacto na saúde dos profissionais de enfermagem frente a COVID-19. Cogitare Enfermagem, 25, e.72702.

Ornell, F., et al. (2020). Pandemia de medo e COVID-19: impacto na saúde mental e possíveis estratégias. Revista Debates in Psychiatry, $10(2), 12-17$.

Oliveira, A.C., Lucas, T.C. \& Iquiapaza, R..A. (2020). What has the COVID-19 pandemic taught us about adopting preventive measures? Texto contexto enfermagem, 29, e.20200106.

Pereira, M.D., et al. (2020). Sofrimento emocional dos Enfermeiros no contexto hospitalar frente à pandemia de COVID-19. Research, Society and Development, 09(8), e.67985121.

Reyes, A.N. \& Fermann, I.L. (2017). Eficácia da terapia cognitivo-comportamental no transtorno de ansiedade generalizada. Revista Brasileira de Terapias Cognitivas, Rio de Janeiro, 13(1), 49-54.

Ribeiro, E.G., Souza, E.L. \& Eler, R. (2020). Saúde Mental na Perspectiva do Enfrentamento à COVID-19: Manejo das Consequências Relacionadas ao Isolamento Social. Rev. Enfermagem e Saúde Coletiva, 04(2), 47-57.

Ribeiro, I.A., et al. (2020). Isolamento social em tempos de pandemia por COVID-19: Impactos na saúde mental da população. Revista Enfermagem Atual In Derme, 92(30).

Rodrigues, N.H. \& Silva, L.G.A. (2020). Gestão da pandemia Coronavírus em um hospital: Relato de experiência profissional. Journal Nursing and Health, 10(4), e.20104004.

Rolim, J.A., Oliveira, A.R. \& Batista, E.C. (2020). Manejo da Ansiedade no Enfrentamento da Covid-19. Rev. Enfermagem e Saúde Coletiva, São Paulo, 04(2), 64-74.

Schmidt, B., et al. (2020). Saúde mental e intervenções psicológicas diante da pandemia do novo coronavírus (COVID-19). Estudos de psicologia, Campinas, 37.

Schuchmann, A.Z., et al. (2020). Isolamento social vertical X Isolamento social horizontal: os dilemas sanitários e sociais no enfrentamento da pandemia de COVID-19. Brazilian Journal of health Review, Curitiba, 03(2), 3556-3576.

Shaw, S.C.K. (2020). Hopelessness, helplessness and resilience: The importance of safeguarding our trainees' mental wellbeing during the COVID-19 pandemic. Nurse Education in Practice, 44, e.102780.

Tan, B.Y.Q., et al. (2020). Psychological Impact of the COVID-19 Pandemic on Health Care Workers in Singapore. Annals of Internal Medicine.

Vasconcelos, C.S.S., et al. (2020). O novo coronavírus e os impactos psicológicos da quarentena. Revista Desafios, 07(Supl.).

Wen, L., et al. (2020). Psychological status of medical workforce during the COVID-19 pandemic: A cross-sectional study. Psychiatry Research, 288, e..112936.

Yuan, W., et al. (2020). A Comparison of Burnout Frequency Among Oncology Physicians and Nurses Working on the Frontline and Usual Wards During the COVID-19 Epidemic in Wuhan, China. Journal of Pain and Symptom Management, 60(1), 60-65.

Zanon, C., et al. (2020). COVID-19: Implicações e aplicações da Psicologia Positiva em tempos de pandemia. Contribuições da psicologia no contexto da pandemia da COVID-19. Estudos de Psicologia, Campinas, 37, e.200072. 